(C) 2020. This manuscript version is made available under the CC-BY-NC-ND 4.0 license http://creativecommons.org/licenses/by-nc-nd/4.0/

\title{
Secretomes of medically important fungi reflect morphological and phylogenetic diversity
}

Sarai Varona ${ }^{a, 1}$, José L. Lavín ${ }^{a, 2, *}$, and José A. Oguiza ${ }^{\text {b, * }}$

a Bioinformatics Unit, CIC bioGUNE-BRTA, Bizkaia Technology Park, 48160 Derio, Bizkaia, Spain

b Genetics and Microbiology Research Group, Institute for Multidisciplinary Research in Applied Biology (IMAB), Universidad Pública de Navarra, 31006 Pamplona, Spain

${ }^{1}$ Present address: Instituto de Salud Carlos III, Unidad de Bioinformática (BU-ISCIII), 28222 Majadahonda, Madrid

2 Present address: Applied Mathematics Department, Bioinformatics Unit, NEIKER, Berreaga Kalea 1, 48160 Derio, Bizkaia, Spain

* Corresponding authors:

José A. Oguiza, Genetics and Microbiology Research Group, Institute for Multidisciplinary Research in Applied Biology (IMAB), Universidad Pública de Navarra, 31006 Pamplona, Spain. Tel: +34 948 169757; E-mail address: jose.oguiza@unavarra.es

José L. Lavín, Applied Mathematics Department, Bioinformatics Unit, NEIKER, Berreaga Kalea 1, 48160 Derio, Bizkaia, Spain. Tel: +34 600004 815; E-mail address: jllavin@neiker.eus

Keywords: Secretome, Mammalian pathogenic fungi, Morphology, Phylogeny, Comparative analysis. 


\section{ABSTRACT}

Secretome represents a main target for understanding the mechanisms of fungal adaptation. In the present study, we focus on the secretomes of fungi associated with infections in humans and other mammals in order to explore relationships between the diverse morphological and phylogenetic groups. Almost all the mammalian pathogenic fungi analyzed have secretome sizes smaller than 1000 proteins and, secreted proteins comprise between $5 \%$ and $10 \%$ of the total proteome. As expected, the correlation pattern between the secretome size and the total proteome was similar to that described in previous secretome studies of fungi. With regard to the morphological groups, minimum secretome sizes of less than 250 secreted proteins and low values for the fraction of secreted proteins are shown in mammalian pathogenic fungi with reduced proteomes such as microsporidia, atypical fungi and some species of yeasts and yeast-like fungi (Malassezia). On the other hand, filamentous fungi have significantly more secreted proteins and the highest numbers are present in species of filamentous fungi that also are plant or insect pathogens (Fusarium verticilloides, Fusarium oxysporum and Basidiobolus meristosporus). With respect to phylogeny, there are also variations in secretome size across fungal subphyla: Microsporidia, Taphrinomycotina, Ustilagomycotina and Saccharomycotina contain small secretomes; whereas larger secretomes are found in Agaricomycotina, Pezizomycotina, Mucoromycotina and Entomophthoromycotina. Finally, principal component analysis (PCA) was conducted on the complete secretomes. The PCA results revealed that, in general, secretomes of fungi belonging to the same morphological group or subphyla cluster together. In conclusion, our results point out that in medically important fungi there is a relationship between the secretome and the morphological group or phylogenetic classification. 


\section{Introduction}

Humans and animals are highly resistant to most invasive infections by fungi (Köhler et al., 2015; Köhler et al., 2017), and only a small number of species in the fungal kingdom can cause human and animal infections (Heitman, 2011; Brown et al., 2012; Gostin ar et al., 2018). Medically important fungi cover true pathogens, commensals and opportunistic pathogens (Sexton and Howlett, 2006; Mayer et al., 2013; Gostin ar et al., 2018). However, a limited proportion of the clinically relevant species of fungi can be considered true pathogens that are able to infect healthy mammal hosts. Commensal fungi typically survive without tissue invasion and can emerge to cause occasional infections. Most opportunistic fungi are environmental species with a saprophytic lifestyle that are not specialized for the mammal host, and usually cause invasive life-threatening systemic infections in immunocompromised hosts (de Hoog et al., 2014). Moreover, many environmental filamentous pathogenic fungi also elicit toxic effects upon ingestion and produce allergic responses (Wanger et al., 2017). Although some fungal pathogens such as Pneumocystis species are directly transmitted between individuals, direct transmission is not usually a regular part of the disease cycle of fungal pathogens (Sexton and Howlett, 2006).

Fungi are exodigesters that require secreted proteins to alter their environment and the organisms they colonize (Girard et al., 2013) and, consequently, secretome represents a main target for understanding the mechanisms of fungal adaptation. Secreted proteins play important roles in the development of fungal diseases in mammalian hosts, enabling invasive infection and nutrient acquisition, establishing interactions with hosts and directly modulating the host immune response (Ranganathan and Garg, 2009; Wartenberg et al., 2011; Lowe and Howlett, 2012). 
Secretome analysis may have clinical applications toward a better control of fungal diseases of humans and animals providing new information for the identification of potential diagnostic biomarkers, therapeutic targets, vaccine development and antifungal strategies (Girard et al., 2013; Sorgo et al., 2013).

Bioinformatic approaches make possible to the large-scale prediction and analysis of the entire set of secreted proteins in a fungal species (Choi et al., 2010; Lum and Min, 2011; Girard et al., 2013; Cortázar et al., 2014; Cortázar et al., 2015). However, up to now there is only limited knowledge about the diversity, composition and evolution of secretomes of fungi that infect humans and other mammals. The rapid accumulation of complete genome sequences now provide us the opportunity to analyze exclusively the predicted secretomes from fungal species associated with mammalian diseases. In this study, we explore relationships between the secretomes and the different morphological and phylogenetic groups of medically important fungi.

\section{Materials and methods}

\subsection{Organisms and sequence data}

To delineate relationships between the secretomes of fungi that are commonly associated with infections in humans and other mammals, publicly available proteomes of fungal species were retrieved from NCBI (as July of 2019). The proteome dataset was composed of 80 representative pathogenic species of the following fungal phyla: Ascomycota, Basidiomycota, Mucoromycota, Zoopagomycota, Microsporidia and Chytridiomycota (Table 1). Only one species per genus was included in the proteome dataset in order to minimize data duplication. The pathogenic fungi analyzed comprised predominantly species belonging to Ascomycota (58 species), followed by 
Basidiomycota (7 species), Microsporidia (7 species), Mucoromycota (5 species), Zoopagomycota (2 species), and Chytridiomycota (1 species). As outgroup species, this proteome dataset contained three fungi that are not pathogenic of mammals: Batrachochytrium dendrobatidis, Encephalitozoon romaleae and Pochonia chlamydosporia. The chytridiomycete $B$. dendrobatidis causes a skin infection in amphibians (Fisher et al., 2012) and currently none of the Chytridiomycota are not known to infect mammals (Köhler et al., 2017). The microsporidia E. romaleae infects grashoppers and its genome is strikingly similar to those of Encephalitozoon species infecting humans (Pombert et al., 2012). Finally, the ascomycete $P$. clamydosporia infects eggs and females of economically important plant-parasitic nematodes (Braga and de Araújo, 2014).

\subsection{Secretome analysis: prediction of secreted proteins}

The secretomes of fungal species (Table S1) were obtained using the pipeline SECRETOOL, a bioinformatic resource for prediction of secreted proteins in fungi (Cortázar et al., 2014). SECRETOOL is a web-based analysis tool (http://genomics.cicbiogune.es/SECRETOOL/Secretool.php) that comprises a group of widely used bioinformatics tools, such as SignalP, TargetP, TMHMM, PredGPI, and WoLF PSORT. Sperschneider et al. (2015) have already evaluated the sensitivity and specificity of these individual bioinformatics tools applied to predictions of secreted proteins in fungi. In addition, Vivek-Ananth et al. (2018) have carried out a comparative analysis of computational pipelines for secretome prediction in fungi, including SECRETOOL. Only soluble secreted proteins through a classical secretion pathway and satisfying the following parameters were analyzed: proteins that possess an $\mathrm{N}$ - 
terminal signal peptide for entering the classical secretion pathway, without the presence of internal transmembrane domains, complete absence of GPI-anchor sites and extracellular secretion of the proteins outside the cell. The identification of secreted proteins using SECRETOOL was designed to be extra stringent, and exclusion of some proteins can occur in the predicted secretomes due to the stringency of the combined default values: SignalP (cut-off 0.8 ), TargetP (cut-off 0.8 ), TMHMM (0 or 1 transmembrane domains), PredGPI (cut-off 0.005), and WoLF PSORT (cut-off 14).

\subsection{Machine learning protocols}

Full sets of secreted proteins in medically important fungi were compared using an all-versus-all BLAST type approach, and a set of unique ortholog proteins was then used to create the reference column to generate the final table. The set of unique ortholog proteins could be defined as a list consisting of all the secreted orthologs, including those that can be found in different species, and those only present in a particular phylum, genera or species, thus, trying to comprise all the possible candidates derived from the predicted secretomes. A set of Perl an R scripts was designed to carry out BLASTp searches to detect putative orthologs (E-value cut-off e-5). BLASTp output was parsed via an in house Perl script, and a presence/absence (P/A) table was generated: the presence of an ortholog of the protein $X$ in the strain $Y$ was represented as "1", while its absence was considered as "0". The P/A table was remodeled and filtered using "unique" function from $\mathrm{R}$ data.table package to enable removal of replicated ortholog relations. The rows of the table represented all the unique secreted proteins and the columns represented the analyzed species. The P/A table was the input for unsupervised machine learning protocol via principal component analysis (PCA) to cluster fungal species based on their secretome content in an 
unsupervised manner so clustering was not biased by any kind of categorization. Data was processed for PCA analysis using R base function "prcomp" for the PCA analysis itself. Then "caret" package was used to remove zero variance variables and "factoextra" package (https://CRAN.R-project.org/package=factoextra) determined dimension and variable contributions, and plotted the corresponding graphics. Threedimensional PCA (PCA 3D) were plotted using "rgl" R package (https://CRAN.Rproject.org/package=rgl).

\section{Results and discussion}

\subsection{Morphological and phylogenetic diversity}

Morphology is an important characteristic used for the differentiation of fungal species, and medically important fungi can be separated into different morphological groups without regard to phylogenetic classification (Sciortino, 2017; Wanger et al., 2017). The principal morphological difference between mammalian pathogenic fungi is that one group grows as yeast or yeast-like single cells, and other group has a multicellular filamentous growth. Moreover, the thermally dimorphic fungi pathogenic to humans and mammals overlap between these two morphological groups, and include Ascomycota species that are capable of temperature dependent alterations in morphological state. A common attribute of all thermally dimorphic fungi is the response to shifts in temperature by transforming from mycelial growth in the environment to yeast cells within the mammalian host (Gauthier, 2017). On the other hand, both atypical fungi and microsporidia are obligate intracellular parasites in humans and animals. Atypical fungi comprises Pneumocystis species with at least two different life cycle forms, the trophic form and the cyst (Ma et al., 2018). Microsporidia 
are highly host dependent fungi with an extreme genome reduction (Han and Weiss, 2017; Wadi and Reinke, 2020).

For the purposes of this study, fungal species that cause infections in mammals were assigned into the above groups based on their morphological characteristics: yeasts and yeast-like fungi, filamentous fungi, thermally dimorphic fungi, atypical fungi, and microsporidia (Table 1). Pathogenic yeasts and yeast-like fungi comprise species of Ascomycota belonging to Saccharomycotina, and Basidiomycota belonging to Ustilagomycotina and Agaromycotina (Table 1). Filamentous fungal pathogens are mainly species of Ascomycota belonging to Pezyzomycotina, except for zygomycete fungi and the basidiomycete Schizophyllum commune. Besides that, the pathogenic filamentous fungi are a broad group of species that can be assorted into different morphological subgroups: dermatophyte fungi, hyaline fungi, dematiaceous fungi and zygomycete fungi (Table 1). Dermatophyte fungi cause a variety of skin diseases in animals and humans. Dermatophyte fungi and thermally dimorphic fungi are closely related Ascomycota, all belonging to Pezizomycotina order Onygenales (Martinez et al., 2012). Hyaline fungi are characterized by narrow, septate and colorless hyphae; dematiaceous fungi contain melanin in their cell wall, which causes the brown and black coloured pigmentation of the septate hyphae; and zygomycete fungi usually form aseptate hyphae (Sciortino, 2017; Wanger et al., 2017). The basidiomycete S. commune hyaline fungus belongs to Agarocomycotina, and is a wood decomposer that has also been demonstrated to be an opportunistic pathogen of humans and animals (Ohm et al., 2010). Dematiaceous fungi are mainly ubiquitous saprophytic fungi found in soil and plants (Chowdhary et al., 2015). Zygomycete fungi are clinically relevant opportunistic species that have been separated into two phyla, the Mucoromycota and Zoopagomycota (Spatafora et al., 2016). The subphylum 
Mucoromycotina of Mucoromycota comprises important opportunistic pathogens of humans and animals (de Hoog et al., 2014), and the subphylum Entomophthoromycotina of Zoopagomycota includes species that cause infection in insects and mammalian hosts (Spatafora et al., 2016; Vilela and Mendoza, 2018). Finally, some morphological groups correlate with their phylogenetic classification: the morphological group of atypical fungi contains only Ascomycota species belonging to Taprinomycotina, and the morphological group of microsporidia only species of the phylum Microsporidia (Table 1).

\subsection{Secretome sizes}

Proteome sizes of the pathogenic fungi analyzed in this study varied significantly ranging from 1831 to 27347 total proteins in Encephalitozoon romaleae and Fusarium oxysporum, respectively (Table 1). In terms of the number of total proteins that were part of the bioinformatically predicted secretome, there was also an extensive variation among the pathogenic fungi investigated: Encephalitozoon species had the smallest secretomes containing 61 to 88 proteins, and the largest were the Fusarium verticilloides, F. oxysporum and Basidiobolus meristosporus secretomes with 1535, 1846 and 1894 proteins, respectively (Table 1). As observed previously (Lowe and Howlett, 2012; Krijger et al., 2014; Lo Presti et al., 2015), for most of the fungal species secreted proteins comprised between $5 \%$ and $10 \%$ of the total proteome (Table 1). The lowest proportion of secreted proteins was present in the grasshopper pathogen E. romaleae (3.3\%), and the highest in the entomopathogen B. meristosporus (11.8\%) (Table 1).

Furthermore, previous studies have revealed that fungal secretome size correlates with lifestyle, with human and animal pathogens having smaller secretomes 
than plant and insect pathogens (Lowe and Howlett, 2012; Sorgo et al., 2013; Krijger et al., 2014; Kim et al., 2016; Le Marquer et al., 2019). The presence of small secretomes in human and animal pathogenic fungi can be related to environments or niches with compounds or nutrients that are easier to obtain and metabolize, and may be a strategy to evade the host immune system (Krijger et al., 2014). In correspondence with this, the majority of the mammalian pathogenic fungi analyzed contained predicted secretome sizes smaller than 1000 proteins (Table 1). Remarkably, only six of the mammalian pathogens were filamentous fungi with predicted secretomes containing more than 1000 proteins: the Pezizomycotina Aspergillus flavus, F. oxysporum, F. verticilloides, and Alternaria alternata, and the Entomophthoromycotina B. meritosporum and Conidiobolus coronatus (Table 1). The presence of high numbers of secreted proteins in fungi is most likely associated with to their pathogenicity in plants or insects (Kim et al., 2016; Le Marquer et al., 2019). Accordingly, the aforementioned Pezizomycotina filamentous fungi are also important plant pathogens and, in addition, $A$. flavus causes infection in insects (St. Leger et al., 2000; Thomma, 2003; Nucci and Anaissie, 2007). The Entomophthoromycotina B. meritosporum and C. coronatus are entomopathogenic fungi that parasitize and kill arthropods (Vilela and Mendoza, 2018).

\subsection{Secretome size and fungal morphology}

Multiple studies have analyzed the repertoires of secreted proteins in fungal species. In general, it has been pointed out that secretome size correlates with proteome size, but also that variations in secretome size can be related with different fungal lifestyles (Lowe and Howlett, 2012; Girard et al., 2013; Sorgo et al., 2013; Meinken et al., 2014; Lo Presti et al., 2015; Kim et al., 2016; Le Marquer et al., 2019). 
However, a comparative analysis including mostly Ascomycota secretomes proposed a correlation of secretome size with phylogeny rather than with fungal lifestyle (Krijger et al., 2014). Besides that, other analyses mainly performed on Basidiomycota supported different hypotheses, such as that secretome size is not related to the fungal lifestyle (Pellegrin et al., 2015) or that secretomes reflect fungal lifestyles rather than phylogenetic classifications (Alfaro et al., 2016). Furthermore, yeasts and yeast-like fungi tend to have considerably reduced numbers of secreted proteins than filamentous fungi suggesting a correlation between secretome size and the complexity of the life cycle (Lowe and Howlett, 2012; Sorgo et al., 2013; Le Marquer et al., 2019).

First, we examined the differences in the number of secreted proteins among the morphological groups of medically important fungi. As expected, the correlation pattern between the secretome size and the total proteome was similar to that observed in other previous studies of fungal secretomes (Lowe and Howlett, 2012; Krijger et al., 2014; Kim et al., 2016) (Fig. 1). In addition, we observed a remarkable variation in the abundance of secreted proteins across morphological groups (Figs. 1 and $2 \mathrm{~A}$ ). In particular, filamentous fungi tended to have significantly more secreted proteins (430-1894) than microsporidia (61-243), atypical fungi (136-147), yeasts and yeast-like fungi (180-591) and thermally dimorphic fungi (381-704) (Fig. 2A; Table 1). The presence of higher numbers of secreted proteins in filamentous fungi may be associated to the filamentous lifestyle.

However, there were not significant variations among the morphological groups for the fraction of predicted proteins that are secreted, with only two exceptions: the Entomophthoromycotina B. meritosporum and C. coronatus, and the mammalian pathogenic fungi with reduced proteomes (below 5000 proteins) (Table 1). B. meritosporum and C. coronatus also cause infection in insects and exhibited the 
highest values for the fraction of secreted proteins with $11.8 \%$ and $10 \%$, respectively (Table 1). On the other hand, minimum secretome sizes (less than 250 secreted proteins) and low values for the fraction of secreted proteins were found in mammalian pathogenic fungi with reduced proteomes (below 5000 proteins) such as microsporidia (3.3-7.6 \%), atypical fungi (3.7-4.1\%) and members of the genus Malassezia (4.3-4.9 \%) (Table 1). Microsporidia and atypical fungi are obligate fungal pathogens depending on the presence of eukaryotic cells to complete their life cycle. Malassezia species are basidiomycete yeasts associated with mammals as superficial commensals that can cause multiple skin disorders. With the exception of Malassezia pachydermatis, all the other Malasezzia species have an obligate lipophilic lifestyle and require fatty acids for growth (Xu et al., 2007; Schuster et al., 2018). Low values for the fraction of secreted proteins might also be related with the adaptation to an obligate intracellular parasitic lifestyle in microsporidia and atypical fungi or to a lipophilic lifestyle in Malassezia yeasts.

Finally, the pathogenic filamentous fungi analyzed are a very large group of species exhibiting considerable variation in their secretome sizes (430-1894) (Fig. 2A; Table 1). In contrast, the subgroup of dermatophyte fungi showed only limited differences in their number of predicted secreted proteins (512-647) (Fig. 2A; Table 1). Dermatophytes are closely phylogenetically related filamentous fungi highly specialized to infect keratinized tissues such as hair, nails and skin (Köhler et al., 2015). Besides, it is also particularly noteworthy that Pseudogymnoascus destructans can be distinguished by the smallest secretome size (430) among the filamentous fungi investigated (Table 1). $P$. destructans is a psychrophilic fungus that grows in a strict temperature range of $\sim 4-20^{\circ} \mathrm{C}$ and is associated with the white-nose syndrome (WNS) skin infection in bats. Although bats are mammal animals, they are susceptible to WNS 
during their hibernation period (Palmer et al., 2018). Likewise $P$. destructans exhibited a largely reduced secretome $(\sim 50 \%)$ in comparison to nonpathogenic Pseudogymnoascus species (Palmer et al., 2018). Altogether, our analysis showed a correlation between secretome size and the morphological groups of medically important fungi (Figs. 1 and 2A).

\subsection{Secretome size and fungal phylogeny}

Krijger et al., (2014) carried out a comparative analysis of 36 fungal and Oomycota secretomes, and proposed that secretome size correlates with phylogeny and to a lesser extent with fungal lifestyle. The wide analysis of 136 fungal species by Kim et al. (2016) revealed a high variation of secretome sizes across phylogenetic lineages. Microsporidia contained the smallest secretomes; and in Ascomycota, species belonging to the subphylum Pezizomycotina had significantly larger secretomes than those of Taphrinomycotina and Saccharomycotina; and in Basidiomycota, species belonging to Pucciniomycotina and Agaricomycotina had larger secretomes than those of Ustilaginomycotina (Kim et al., 2016). With regard to the mammalian pathogenic fungi compared here, we have also observed similar variations in secretome size across fungal subphyla: small secretomes were present in Microsporidia (61-243), Taphrinomycotina (136-147), Ustilagomycotina (180-222) and Saccharomycotina (263-463). Whereas larger secretomes with a considerably size variation were found in Agaricomycotina (342-891), Pezizomycotina (381-1846), Mucoromycotina (628-865) and Entomophthoromycotina (1061-1894) (Fig. 2B; Table 1). Accordingly, our analysis indicated that there is also a relationship between the secretome size and the phylogenetic classification of medically important fungi. 


\subsection{Principal component analysis}

To corroborate the relationships based on secretome sizes, principal component analysis (PCA) was performed to compare the complete secretomes (Figs. 3 and S1). Our expectation was to observe clustering of secretomes according to morphology and phylogenetic classification of mammalian pathogenic fungi. As a result, PCA highlighted that secretomes of species belonging to the same morphological group or subphyla cluster together, excepting the three species of filamentous fungi with the largest secretomes: the entomopathogen B. meristosporus, and the plant pathogens F. oxysporum and F. verticilloides (Figs. 3 and Fig. S1). With respect to morphological groups, secretomes of yeasts and yeast-like fungi, thermally dimorphic fungi, atypical fungi, and microsporidia formed separate clusters in the PCA; whereas secretomes of filamentous fungi did not grouped together and occupied distinct positions. Noteworthy, secretomes of each morphological subgroup of filamentous fungi (dermatophyte fungi, hyaline fungi, dematiaceous fungi and zygomycete fungi) were arranged in separate clusters (Figs. 3A and S1).

\section{Conclusion}

Secreted proteins play important roles in the interaction of a fungal pathogen with its mammalian host. In the present study, we bioinformatically predicted and compared the secretomes of fungi that infect humans and other mammals. Among the clinically relevant fungi analyzed, there was an extensive variation of secretome sizes (61-1894 secreted proteins), although in almost all the species secretome sizes were smaller than 1000 proteins. Only some species of filamentous fungi that also are plant or insect pathogens showed considerably larger secretomes. Overall, our results are in accordance with previous studies of fungal secretomes stating the correlation 
between the secretome size and the total proteome (Lowe and Howlett, 2012; Krijger et al., 2014; Kim et al., 2016). In addition, our observations point out that in medically important fungi there is a relationship between the secretome and the morphological group or phylogenetic classification. However, at present it is not possible to define whether the morphology, the phylogeny or the lifestyle produces the strongest effect on the secretomes of fungal species that cause infections in mammals. Further functional and comparative secretome analyses are needed to advance in the understanding of the role of secreted proteins in the mammalian pathogenic lifestyle of fungi. 


\section{Conflicts of interest}

The authors state that there are no conflicts of interest related to this publication.

\section{Acknowledgments}

This work was supported by research project RTI2018-099371-B-I00 of the

Spanish National Research Plan and by additional institutional support from the Public University of Navarre, and the $\mathrm{MCl}$ for the Severo Ochoa Excellence accreditation (SEV-2016-0644).

\section{Appendix A. Supplementary data}

The following are the Supplementary data to this article:

TableS1.xlsx Table S1. Secretomes of medically important fungi

FigureS1.7z Figure S1. PCA 3D of the secretomes of medically important fungi 


\section{References}

Alfaro, M., Castanera, R., Lavín, J.L., Grigoriev, I.V., Oguiza, J.A., Ramírez, L., Pisabarro, A.G., 2016. Comparative and transcriptional analysis of the predicted secretome in the lignocellulose-degrading basidiomycete fungus Pleurotus ostreatus. 2016. Environ. Microbiol. 18, 4710-4726.

Braga, F.R., de Araújo, J.V., 2014. Nematophagous fungi for biological control of gastrointestinal nematodes in domestic animals. Appl. Microbiol. Biotechnol. 98, 71-82.

Brown, N.A., Antoniw, J., Hammond-Kosack, K.E., 2012. The predicted secretome of the plant pathogenic fungus Fusarium graminearum: a refined comparative analysis. PLoS One 7, e33731.

Choi, J., Park, J., Kim, D., Jung, K., Kang, S., Lee, Y.H., 2010. Fungal Secretome Database: Integrated platform for annotation of fungal secretomes. BMC Genomics $11,105$.

Chowdhary, A., Perfect, J., de Hoog, G.S., 2015. Black molds and melanized yeasts pathogenic to humans. Cold Spring Harb. Perspect. Med. 5, a019570.

Cortázar, A.R., Aransay, A.M., Alfaro, M., Oguiza, J.A., Lavín, J.L., 2014. SECRETOOL: integrated secretome analysis tool for fungi. Amino Acids 46, 471473.

Cortázar, A.R., Oguiza, J.A., Aransay, A.M., Lavín, J.L., 2015.PECAS: prokaryotic and eukaryotic classical analysis of secretome. Amino Acids 47, 2659-2663.

de Hoog, S., Ibrahim, A.S., Voigt, K., 2014. Zygomycetes: an emerging problem in the clinical laboratory. Mycoses 57 Suppl. 3, 1. 
Fisher, M., Henk, D., Briggs, C., Brownstein, J.S., Madoff, L.C., McCraw, S.L., Gurr, S.J., 2012. Emerging fungal threats to animal, plant and ecosystem health. Nature 484, 186-194.

Gauthier, G.M., 2017. Fungal dimorphism and virulence: molecular mechanisms for temperature adaptation, immune evasion, and in vivo survival. Mediators Inflamm. 2017,8491383

Girard, V., Dieryckx, C., Job, C., Job, D., 2013. Secretomes: the fungal strike force. Proteomics 13, 597-608.

Gostin ar, C., Zajc, J., Lenassi, M., Plemenitas, A., de Hoog, S., Al-Hatmi, A.M.S., Gunde-Cimerman, N., 2018. Fungi between extremotolerance and opportunistic pathogenicity on humans. Fungal Diversity 93, 195-213.

Han, B., Weiss, L.M., 2017. Microsporidia: obligate intracellular pathogens within the fungal kingdom. Microbiol. Spectrum 5, FUNK-0018-2016.

Heitman, J., 2011. Microbial Pathogens in the Fungal Kingdom. Fungal Biol. Rev. 25, 48-60.

Kim, K.T., Jeon, J., Choi, J., Cheong, K., Song, H., Choi, G., Kang, S., Lee, Y.H., 2016. Kingdom-wide analysis of fungal small secreted proteins (SSPs) reveals their potential role in host association. Front. Plant. Sci. 7, 186.

Köhler, J.R., Casadevall, A., Perfect, J., 2015. The spectrum of fungi that infects humans. Cold Spring Harb. Perspect. Med. 5, a019273.

Köhler, J.R., Hube, B., Puccia, R., Casadevall, A., Perfect, J.R., 2017. Fungi that infect humans. Microbiol. Spectrum 5, FUNK-0014-2016.

Krijger, J., Thon, M.R., Deising, H.B., Wirsel, S.G., 2014. Compositions of fungal secretomes indicate a greater impact of phylogenetic history than lifestyle adaptation. BMC Genomics 15, 722. 
Le Marquer, M., San Clemente, H., Roux, C., Savelli, B., Frei Dit Frey, N., 2019. Identification of new signalling peptides through a genome-wide survey of 250 fungal secretomes. BMC Genomics 20, 64.

Lo Presti, L., Lanver, D., Schweizer, G., Tanaka, S., Liang, L., Tollot, M., Zuccaro, A., Reissmann, S., Kahmann, R., 2015. Fungal effectors and plant susceptibility. Annu. Rev. Plant Biol. 66, 513-545.

Lowe, R.G.T., Howlett, B.J., 2012. Indifferent, affectionate, or deceitful: lifestyles and secretomes of fungi. PLoS Pathog. 8, e1002515.

Lum, G., Min, X.J., 2011. FunSecKB: the Fungal Secretome KnowledgeBase. Database (Oxford) 2011, bar001.

Ma, L., Cissé, O.H., Kovacs, J.A., 2018. A molecular window into the biology and epidemiology of Pneumocystis spp. Clin. Microbiol. Rev. 31, e00009-18.

Martinez, D.A., Oliver, B.G., Gräser, Y., Goldberg, J.M., Li, W., Martinez-Rossi, N.M., Monod, M., Shelest, E., Barton, R.C., Birch, E., Brakhage, A.A., Chen, Z., Gurr, S.J., Heiman, D., Heitman, J., Kosti, I., Rossi, A., Saif, S., Samalova, M., Saunders, C.W., Shea, T., Summerbell, R.C., Xu, J., Young, S., Zeng, Q., Birren, B.W., Cuomo, C.A., White, T.C., 2012. Comparative genome analysis of Trichophyton rubrum and related dermatophytes reveals candidate genes involved in infection. mBio 3, e00259-12.

Mayer, F.L., Wilson, D., Hube, B., 2013. Candida albicans pathogenicity mechanisms. Virulence 4, 119-128.

Meinken, J., Asch, D.K., Neizer-Ashun, K.A., Chang, G.H, Cooper Jr., C.R., Min, X.J., 2014. FunSecKB2: a fungal protein subcellular location knowledgebase. Comput. Mol. Biol. 4, 1-17. 
Nucci, M., Anaissie, E., 2007. Fusarium infections in immunocompromised patients. Clin. Microbiol. Rev. 20, 695-704.

Ohm, R.A., de Jong, J.F., Lugones, L.G., Aerts, A., Kothe, E., Stajich, J.E., de Vries, R.P., Record, E., Levasseur, A., Baker, S.E., Bartholomew, K.A., Coutinho, P.M., Erdmann, S., Fowler, T.J., Gathman, A.C., Lombard, V., Henrissat, B., Knabe, N., Kües, U., Lilly, W.W., Lindquist, E., Lucas, S., Magnuson, J.K., Piumi, F., Raudaskoski, M., Salamov, A., Schmutz, J., Schwarze, F.W., vanKuyk, P.A., Horton, J.S., Grigoriev, I.V., Wösten, H.A., 2010. Genome sequence of the model mushroom Schizophyllum commune. Nat. Biotechnol. 28, 957-963.

Palmer, J.M., Drees, K.P., Foster, J.T., Lindner, D.L., 2018. Extreme sensitivity to ultraviolet light in the fungal pathogen causing white-nose syndrome of bats. Nat. Commun. 9, 35.

Pellegrin, C., Morin, E., Martin, F.M., Veneault-Fourrey, C., 2015. Comparative analysis of secretomes from ectomycorrhizal fungi with an emphasis on smallsecreted proteins. Front. Microbiol. 6, 1278.

Pombert, J.F., Selman, M., Burki, F., Bardell, F.T., Farinelli, L., Solter, L.F., Whitman, D.W., Weiss, L.M., Corradi, N., Keeling P.J., 2012. Gain and loss of multiple functionally related, horizontally transferred genes in the reduced genomes of two microsporidian parasites. Proc. Natl. Acad. Sci. U.S.A. 109, 12638-12643.

Ranganathan, S., Garg, G., 2009. Secretome: clues into pathogen infection and clinical applications. Genome Med. 1, 113.

Schuster, M., Schweizer, G., Kahmann, R., 2018. Comparative analyses of secreted proteins in plant pathogenic smut fungi and related basidiomycetes. Fungal Genet. Biol. 112, 21-30. 
Sciortino Jr., C.R., 2017. Atlas of Clinically Important Fungi, 1st edn. John Wiley \& Sons, Hoboken, New Jersey.

Sexton, A.C., Howlett, B.J., 2006. Parallels in fungal pathogenesis on plant and animal hosts. Eukaryot. Cell 5, 1941-1949.

Sorgo, A.G., Heilmann, C.J., Brul, S., de Koster, C.G., Klis, F.M., 2013. Beyond the wall: Candida albicans secret(e)s to survive. FEMS Microbiol. Lett. 338, 10-17.

Spatafora, J.W., Chang, Y., Benny, G.L., Lazarus, K., Smith, M.E., Berbee, M.L., Bonito, G., Corradi, N., Grigoriev, I., Gryganskyi, A., James, T.Y., O’Donnell, K., Roberson, R.W., Taylor, T.N., Uehling, J., Vilgalys, R., White, M.M., Stajich, J.E., 2016. A phylum-level phylogenetic classification of zygomycete fungi based on genome-scale data. Mycologia 108, 1028-1046.

Sperschneider, J., Williams, A.H., Hane, J.K., Singh, K.B., Taylor, J.M., 2015. Evaluation of Secretion Prediction Highlights Differing Approaches Needed for Oomycete and Fungal Effectors. Front. Plant Sci. 6, 1168.

St. Leger, R.J., Screen, S.E., Shams-Pirzadeh, B., 2000. Lack of host specialization in Aspergillus flavus. Appl. Environ. Microbiol. 66, 320-324.

Thomma, B.P., 2003. Alternaria spp.: from general saprophyte to specific parasite. Mol. Plant Pathol. 4, 225-236.

Vilela, R., Mendoza, L., 2018. Human pathogenic Entomophthorales. Clin. Microbiol. Rev. 31, e00014-18.

Vivek-Ananth, R.P., Mohanraj, K., Vandanashree, M., Jhingran, A., Craig, J.P., Samal, A., 2018. Comparative systems analysis of the secretome of the opportunistic pathogen Aspergillus fumigatus and other Aspergillus species. Sci. Rep. 8, 6617. 
Wadi, L., Reinke, A.W., 2020. Evolution of microsporidia: An extremely successful group of eukaryotic intracellular parasites. PLoS Pathog. 16, e1008276.

Wanger, A., Chavez, V., Huang, R.S.P., Wahed, A., Actor, J.K., Dasgupta, A., 2017. Microbiology and Molecular Diagnosis in Pathology, 1st edn. Elsevier, Amsterdam.

Wartenberg, D., Lapp, K., Jacobsen, I.D., Dahse, H.M., Kniemeyer, O., Heinekamp, T., Brakhage, A.A., 2011. Secretome analysis of Aspergillus fumigatus reveals Asphemolysin as a major secreted protein. Int. J. Med. Microbiol. 301, 602-611.

Xu, J., Saunders, C.W., Hu, P., Grant, R.A., Boekhout, T., Kuramae, E.E., Kronstad, J.W., Deangelis, Y.M., Reeder, N.L., Johnstone, K.R., Leland, M., Fieno, A.M., Begley, W.M., Sun, Y., Lacey, M..P, Chaudhary, T., Keough, T., Chu, L., Sears, R., Yuan, B., Dawson Jr., T.L., 2007. Dandruff-associated Malassezia genomes reveal convergent and divergent virulence traits shared with plant and human fungal pathogens. Proc. Natl. Acad. Sci. U.S.A. 104, 18730-18735. 


\section{Figure captions}

Fig.1. Correlation between the sizes of the secretome and the total proteome in medically important fungi. Points are coloured according to the classification to morphological groups of the analyzed species.

Fig. 2. Number of secreted proteins with regard to morphological group and phylogenetic classification of medically important fungi. (A) The number of secreted proteins among species in different morphological groups and subgroups. Points are coloured by phylum. (B) The number of secreted proteins among species in different fungal phyla and subphyla. Points are coloured by morphological group. The phylum Chytridiomycota, represented by only one species (Batrachochytrium dendrobatidis), is not included. Sac, Saccharomycotina; Pez, Pezyzomycotina; Tap, Taphrinomycotina; Aga, Agaromycotina; Ust, Ustilagomycotina; Muc, Mucoromycotina; Ent, Entomophthoromycotina.

Fig. 3. Principal component analysis (PCA) of the secretomes of medically important fungi. The PCA scores plot for PC1 and PC2 are shown in (A) with points coloured by morphological group or subgroup, and (B) with points coloured by phylum or subphylum. The percentage of the total variance explained by each principal component is shown in brackets on each axis. Additional information of PCA analysis in Supplementary material, Fig. S1. 
Table 1

Fungal species analyzed in this study.

\section{Species \\ YEASTS AND YEAST-LIKE FUNGI}

Candida glabrata CBS 138

Candida orthopsilosis Co 90-125

Candida tropicalis MYA-3404

Candida dubliniensis CD36

Candida albicans SC5314

Lodderomyces elongisporus NRRL YB-4239

Meyerozyma guilliermondii ATCC 6260

Clavispora lusitaniae ATCC 42720

Malassezia sympodialis ATCC 42132

Malassezia globosa CBS 7966

Malassezia pachydermatis CBS 1879

Cryptococcus neoformans var. neoformans JEC21

Cryptococcus gattii WM276

Trichosporon asahii var. asahii CBS 2479

FILAMENTOUS FUNGI

\section{Dermatophyte fungi}

Trichophyton tonsurans CBS 112818

Trichophyton equinum CBS 127.97

Trichophyton soudanense CBS 452.61

Trichophyton rubrum CBS 118892

Nannizzia gypsea CBS 118893

Microsporum canis CBS 113480

Trichophyton verrucosum HKI 0517

Trichophyton benhamiae CBS 112371

Hyaline fungi

Aspergillus niger ATCC 1015

Aspergillus parasiticus SU-1

Aspergillus flavus NRRL 3357

Aspergillus nidulans FGSC A4

Aspergillus terreus $\mathrm{NIH} 2624$

Aspergillus fumigatus A1163

Aspergillus clavatus NRRL 1

Fusarium oxysporum f. sp. lycopersici 4287

Fusarium verticillioides 7600

Chaetomium globosum CBS 148.51

Pochonia chlamydosporia 170

Scedosporium apiospermum IHEM 14462

Lomentospora prolificans $\mathrm{JHH}-5317$

Pseudogymnoascus destructans 20631-21

Madurella mycetomatis mm55

Schizophyllum commune $\mathrm{H} 4-8$

Dematiaceous fungi

Exophiala dermatitidis NIH/UT8656

Exophiala aquamarina CBS 119918

Exophiala oligosperma CBS 72588

Exophiala spinifera CBS 89968

Cladophialophora bantiana CBS 173.52

Cladophialophora carrionii CBS 160.54

Fonsecaea monophora CBS 269.37

Fonsecaea multimorphosa CBS 102226

Fonsecaea nubica CBS 269.64

Fonsecaea pedrosoi CBS 271.37

Verruconis gallopava CBS 43764

Cyphellophora europaea CBS 101466

Rhinocladiella mackenziei CBS 650.93

Alternaria alternata SRC1IrK2f

Phylum

Subphylum

Proteome

Secretome

$\%$ Proteome

Ascomycota

Ascomycota

Ascomycota

Ascomycota

Ascomycota

Ascomycota

Ascomycota

Ascomycota

Basidiomycota

Basidiomycota

Basidiomycota

Basidiomycota

Basidiomycota

Basidiomycota

Ascomycota
Ascomycota
Ascomycota
Ascomycota
Ascomycota
Ascomycota
Ascomycota
Ascomycota

Ascomycota

Ascomycota

Ascomycota

Ascomycota

Ascomycota

Ascomycota

Ascomycota

Ascomycota

Ascomycota

Ascomycota

Ascomycota

Ascomycota

Ascomycota

Ascomycota

Ascomycota

Basidiomycota

Ascomycota

Ascomycota

Ascomycota

Ascomycota

Ascomycota

Ascomycota

Ascomycota

Ascomycota

Ascomycota

Ascomycota

Ascomycota

Ascomycota

Ascomycota

Ascomycota
Saccharomycotina

Saccharomycotina

Saccharomycotina

Saccharomycotina

Saccharomycotina

Saccharomycotina

Saccharomycotina

Saccharomycotina

Ustilaginomycotina

Ustilaginomycotina

Ustilaginomycotina

Agaricomycotina

Agaricomycotina

Agaricomycotina

Pezizomycotina

Pezizomycotina

Pezizomycotina

Pezizomycotina

Pezizomycotina

Pezizomycotina

Pezizomycotina

Pezizomycotina

Pezizomycotina

Pezizomycotina

Pezizomycotina

Pezizomycotina

Pezizomycotina

Pezizomycotina

Pezizomycotina

Pezizomycotina

Pezizomycotina

Pezizomycotina

Pezizomycotina

Pezizomycotina

Pezizomycotina

Pezizomycotina

Pezizomycotina

Agaricomycotina

Pezizomycotina

Pezizomycotina

Pezizomycotina

Pezizomycotina

Pezizomycotina

Pezizomycotina

Pezizomycotina

Pezizomycotina

Pezizomycotina

Pezizomycotina

Pezizomycotina

Pezizomycotina

Pezizomycotina

Pezizomycotina

$\begin{array}{lll}5202 & 263 & 5.1 \\ 5678 & 338 & 6.0 \\ 6254 & 463 & 7.4 \\ 5860 & 397 & 6.8 \\ 6030 & 414 & 6.9 \\ 5799 & 313 & 5.4 \\ 5920 & 321 & 5.4 \\ 5936 & 302 & 5.1 \\ 4502 & 222 & 4.9 \\ 4286 & 200 & 4.7 \\ 4202 & 180 & 4.3 \\ 6863 & 361 & 5.3 \\ 6565 & 342 & 5.2 \\ 8311 & 591 & 7.1\end{array}$

8521

6.6

$8676 \quad 557 \quad 6.4$

$10671 \quad 643 \quad 6.0$

$10418 \quad 614 \quad 5.9$

$\begin{array}{lll}8921 & 647 & 7.3\end{array}$

$\begin{array}{lll}8765 & 587 & 6.7\end{array}$

$\begin{array}{lll}8028 & 512 & 6.4\end{array}$

$\begin{array}{lll}7405 & 506 & 6.8\end{array}$

$\begin{array}{lll}10950 & 841 & 7.7\end{array}$

$\begin{array}{lll}8645 & 857 & 9.9\end{array}$

$13485 \quad 1146 \quad 8.5$

$9561 \quad 795 \quad 8.3$

$\begin{array}{lll}10401 & 892 & 8.6\end{array}$

$\begin{array}{lll}9929 & 744 & 7.5\end{array}$

$\begin{array}{lll}9121 & 703 & 7.7\end{array}$

$\begin{array}{lll}27347 & 1846 & 6.8\end{array}$

$20553 \quad 1535 \quad 7.5$

$11048 \quad 925 \quad 8.4$

$14204 \quad 1227 \quad 8,6$

$\begin{array}{lll}8376 & 734 & 8.8\end{array}$

$\begin{array}{lll}8560 & 774 & 9.0\end{array}$

$\begin{array}{lll}9153 & 430 & 4.7\end{array}$

$\begin{array}{lll}10707 & 999 & 9.3\end{array}$

$\begin{array}{lll}13194 & 891 & 6.8\end{array}$

$\begin{array}{lll}9578 & 525 & 5.5\end{array}$

$\begin{array}{lll}13118 & 754 & 5.7\end{array}$

$13234 \quad 745 \quad 5.6$

$\begin{array}{lll}12049 & 715 & 5.9\end{array}$

$12762 \quad 770 \quad 6.0$

$10373 \quad 617 \quad 5.9$

$11984 \quad 736 \quad 6.1$

$\begin{array}{lll}12369 & 789 & 6.4\end{array}$

$11681 \quad 748 \quad 6,4$

$\begin{array}{lll}12527 & 772 & 6.2\end{array}$

$11357 \quad 631 \quad 5.6$

$11094 \quad 767 \quad 6.9$

$11382 \quad 627 \quad 5.5$

$\begin{array}{lll}13466 & 1251 & 9.3\end{array}$ 


\section{Zygomycete fungi}

Lichtheimia corymbifera JMRC:FSU:9682

Rhizopus delemar RA 99-880

Rhizopus microsporus ATCC 52813

Mucor circinelloides CBS 277.49

Syncephalastrum racemosum NRRL 2496

Basidiobolus meristosporus CBS 931.73

Conidiobolus coronatus NRRL 28638

\section{THERMALLY DIMORPHIC FUNGI}

Coccidioides posadasii RMSCC 3488

Coccidioides immitis RS

Histoplasma capsulatum NAm1

Paracoccidioides lutzii Pb01

Paracoccidioides brasiliensis $\mathrm{Pb03}$

Blastomyces gilchristii SLH14081

Blastomyces dermatitidis ER-3

Sporothrix schenckii 1099-18

Talaromyces marneffei ATCC 18224

Sporothrix brasiliensis 5110

\section{ATYPICAL FUNGI}

Pneumocystis jirovecii RU7

Pneumocystis murina B123

Pneumocystis carinii B80

\section{MICROSPORIDIA}

Encephalitozoon intestinalis ATCC 50506 Encephalitozoon hellem ATCC 50504

Encephalitozoon romaleae SJ-2008

Encephalitozoon cuniculi GB-M1

Enterocytozoon bieneusi $\mathrm{H} 348$

Vittaforma corneae ATCC 50505

Trachipleistophora hominis

CHYTRIDIOMYCETE FUNGI

\begin{tabular}{lllll} 
Mucoromycota & Mucoromycotina & 13404 & 865 & 6.5 \\
Mucoromycota & Mucoromycotina & 17459 & 821 & 4.7 \\
Mucoromycota & Mucoromycotina & 10891 & 651 & 6.0 \\
Mucoromycota & Mucoromycotina & 11709 & 628 & 5.4 \\
Mucoromycota & Mucoromycotina & 11121 & 722 & 6.5 \\
Zoopagomycota & Entomophthoromycotina & 16110 & 1894 & 11.8 \\
Zoopagomycota & Entomophthoromycotina & 10572 & 1061 & 10.0 \\
& & & & \\
Ascomycota & Pezizomycotina & 9964 & 493 & 4.9 \\
Ascomycota & Pezizomycotina & 9910 & 546 & 5.5 \\
Ascomycota & Pezizomycotina & 9313 & 381 & 4.1 \\
Ascomycota & Pezizomycotina & 8826 & 402 & 4.6 \\
Ascomycota & Pezizomycotina & 8427 & 394 & 4.7 \\
Ascomycota & Pezizomycotina & 11343 & 614 & 5.4 \\
Ascomycota & Pezizomycotina & 11539 & 628 & 5.4 \\
Ascomycota & Pezizomycotina & 10293 & 673 & 6.5 \\
Ascomycota & Pezizomycotina & 10638 & 704 & 6.6 \\
Ascomycota & Pezizomycotina & 9091 & 592 & 6.5 \\
Ascomycota & Taphrinomycotina & 3761 & 143 & 3.8 \\
Ascomycota & Taphrinomycotina & 3623 & 147 & 4.1 \\
Ascomycota & Taphrinomycotina & 3646 & 136 & 3.7 \\
Microsporidia & n.a. & & & \\
Microsporidia & n.a. & 1939 & 80 & 4.1 \\
Microsporidia & n.a. & 1928 & 74 & 3.8 \\
Microsporidia & n.a. & 1831 & 61 & 3.3 \\
Microsporidia & n.a. & 2120 & 88 & 4.2 \\
Microsporidia & n.a. & 3632 & 141 & 3.9 \\
Microsporidia & n.a. & 2239 & 122 & 5.4 \\
Chytridiomycota & n.a. & 3212 & 243 & 7.6 \\
& & & & \\
& & & & 10.4 \\
\hline
\end{tabular}


Figure 1

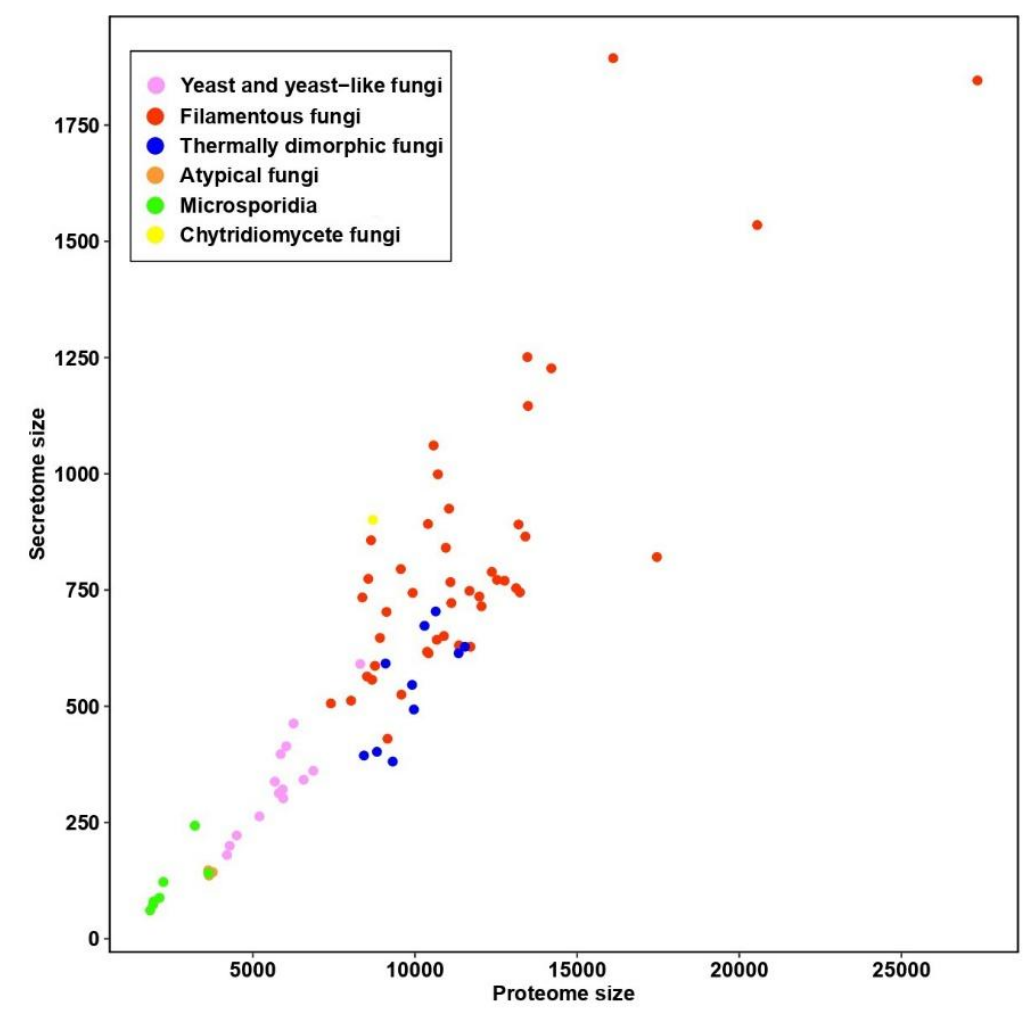


A

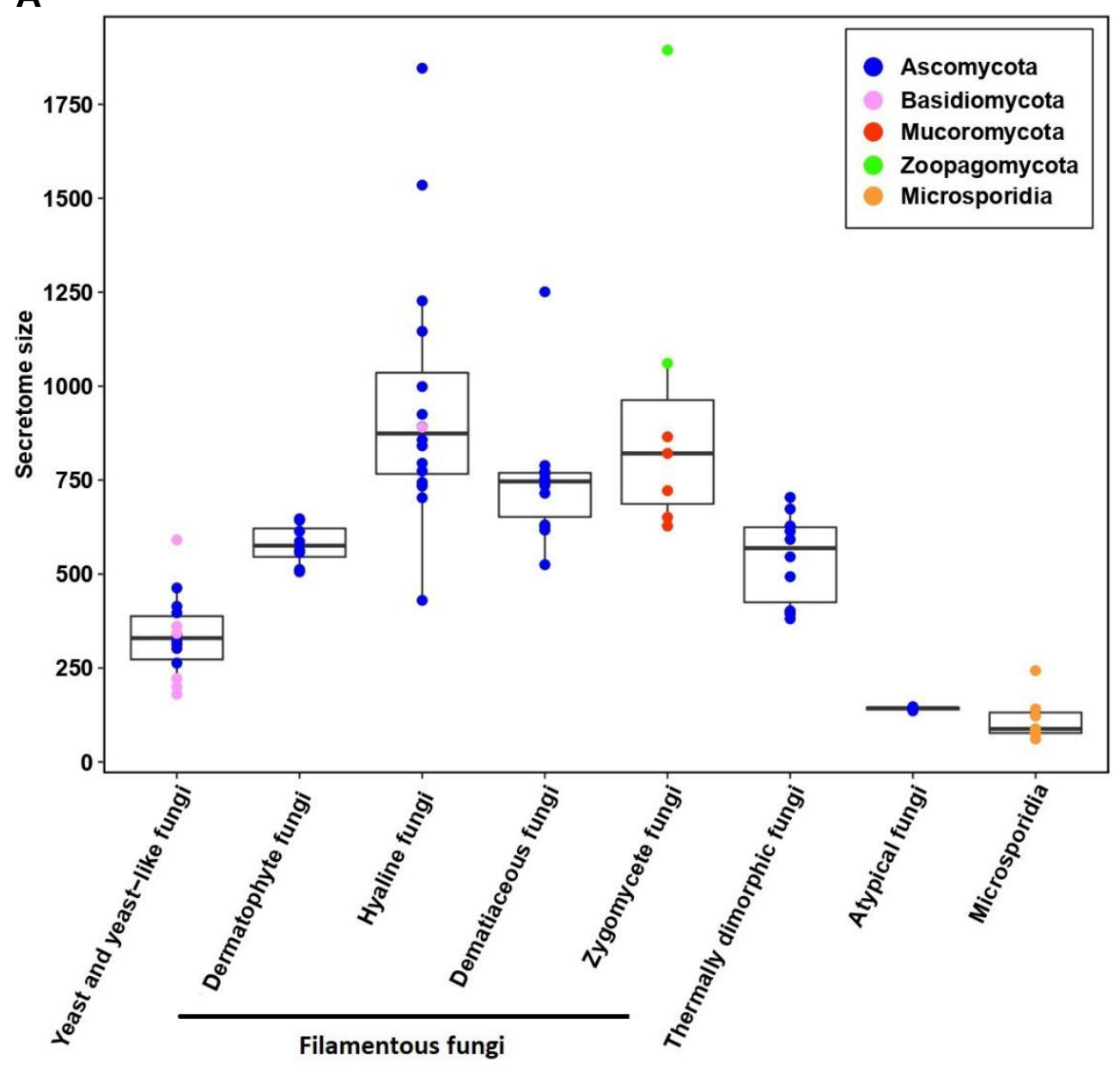

B

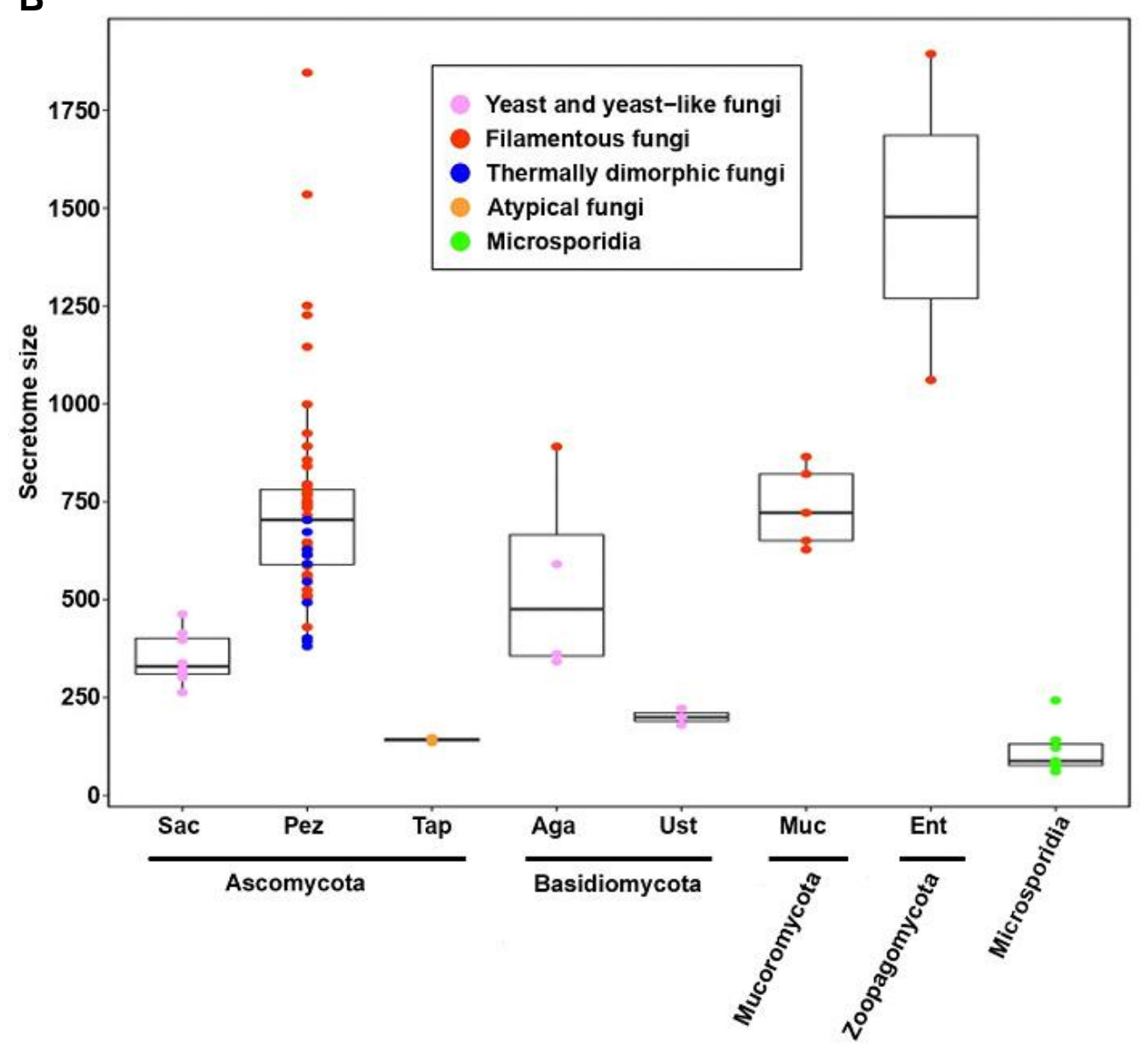
A 
A

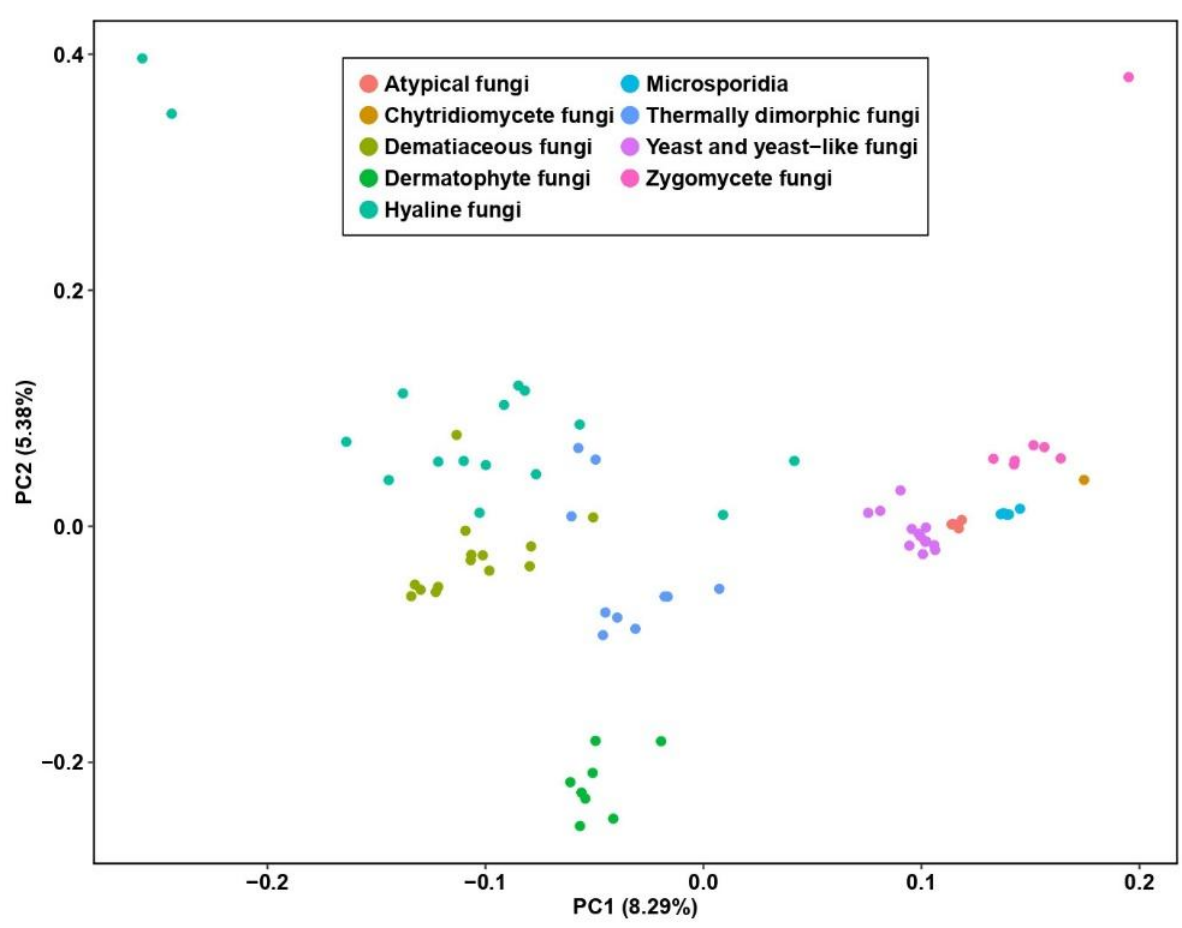

B

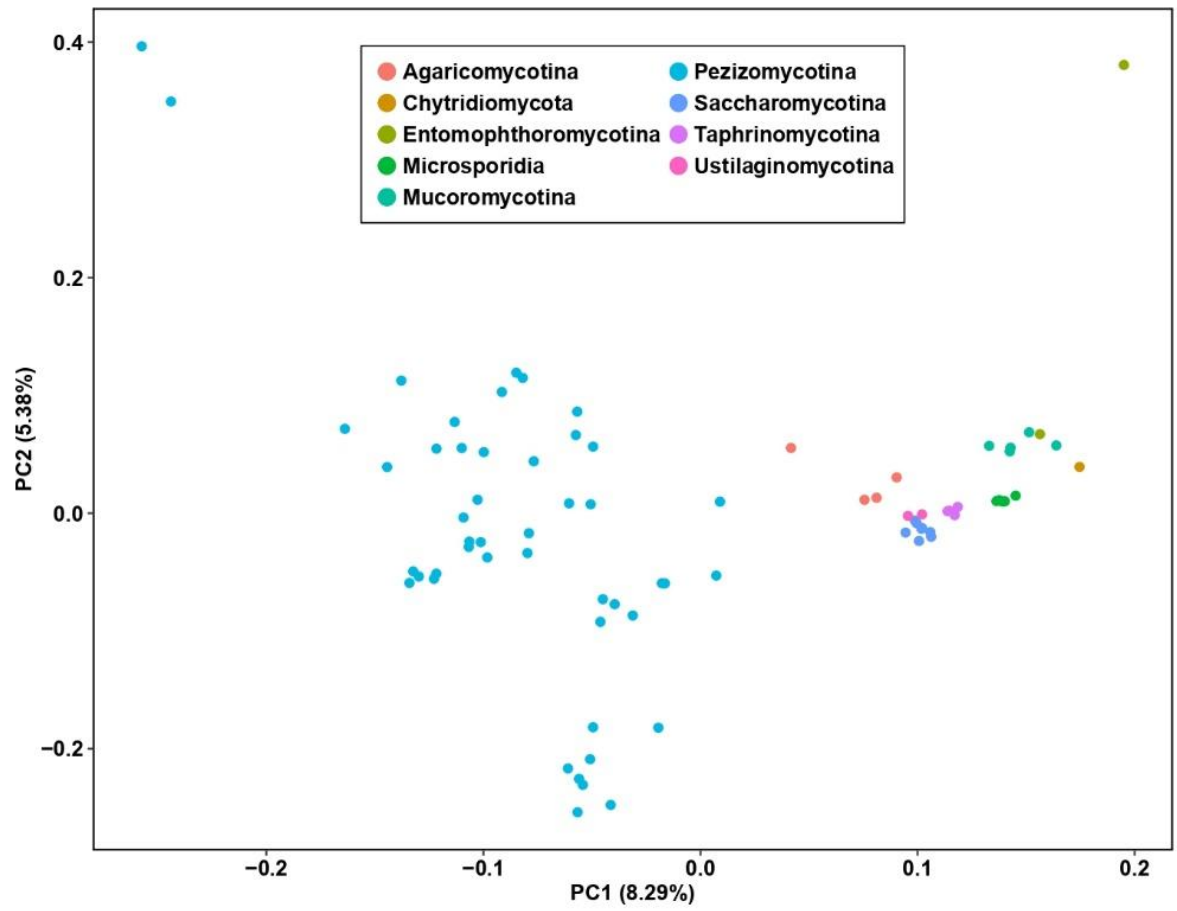

